\title{
LiF 分子的极化对有机发光二极管磁效应的影响
}

\author{
白江文 ${ }^{(1)}$, 张巧明 ${ }^{(1)}$, 雷衍连 ${ }^{(1)}$, 张勇 ${ }^{(1)}$, 熊祖洪 ${ }^{(12 *}$ \\ (1) 西南大学物理科学与技术学院, 发光与实时分析教育部重点实验室, 重庆 400715; \\ (2) 复旦大学应用表面物理国家重点实验室, 上海 200433 \\ *联系人, E-mail: zhxiong@ @ swu.edu.cn \\ 2012-11-18 收稿, 2013-04-22 接受 \\ 重庆市科委自然科学基金(CSTC, 2010BA6002)、复旦大学应用表面物理国家重点实验室开放课题(KL2011_06)、国家自然科学基金 \\ (11374242,11204247)和中央高校基本科研业务费专项资金(XDJK2011C041)资助
}

\begin{abstract}
摘要 在常规的有机发光二极管的发光层中掺入 lithium fluoride (LiF), 可以有效调控器件在 不同温度下的电流与电致发光的磁场效应. 与未掺杂的参考器件相比, $\mathrm{LiF}$ 掺杂器件的磁场效 应的线型与幅度都发生了明显的改变. 在室温下, 当外加磁场强度达到几百毫特斯拉时, 常规 器件的磁场效应随磁场增加缓慢增大并趋于饱和, 而掺杂器件的磁场效应随磁场增加几乎呈 直线增加, 且表现出了不饱和的高场效应. 此外, 随 $\mathrm{LiF}$ 掺杂浓度的增大, 电流与发光的高场 效应的幅度均表现出先增加后降低的变化趋势. 掺杂器件与未掺杂器件磁场效应的差异, 可 以归结为 $\mathrm{LiF}$ 颗粒在器件内电场中发生介电极化的结果. 发光层中的激子与 $\mathrm{LiF}$ 颗粒表面的极 化电荷发生反应, 导致了器件特殊的磁场效应。
\end{abstract}

\section{关键词}

磁电导

极化

三重态激子

激子-电荷反应
自 2003 年 Kalinowski 等人 ${ }^{[1]}$ 在基于 tris-(8-hydroxyquinoline)-aluminum (III) $\left(\mathrm{Alq}_{3}\right)$ 的有机发光二极管 (Orangic light emitting diodes, OLEDs)中发现外加磁 场可以改变有机发光器件的注人电流和电致发光的 强度 (分别称为磁电导和磁电致发光, 即 MC 和 MEL) 之后, 这一现象便引起了研究人员的极大兴趣. 近些 年, 国内外各研究组在有机发光二极管的磁效应这 一领域展开了深人研究 ${ }^{[2 \sim 8]}$, 针对该磁效应的物理机 制提出了多种可能的解释, 例如超精细相互作用机 制 $^{[1]}$ 、双极化子机制 ${ }^{[9]}$ 、激子-电荷反应机制 ${ }^{[10]}$ 、三重 态激子淬灭机制 ${ }^{[11]}$ 等. 但是, 迄今为止还没有一个 系统的理论能完全解释实验发现的所有现象. 在物 理学中, 常用通过改变实验条件和实验对象探究实 验结果产生的变化来揭示某一物理规律. 具体到有 机磁效应的研究中, 可通过设计一些特殊的器件结 构来探究磁效应的变化规律, 进而佐证或完善现有 的磁效应机制. 通常来讲, 这些特殊的器件结构主要

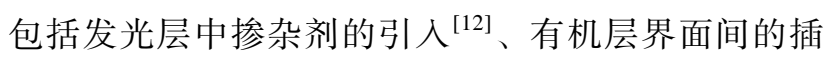

层 $^{[13,14] \text { 、界面的修饰 }}{ }^{[15]}$ 等, 而其中插层和掺杂是常用 的方法.

Odaka 等人 ${ }^{[13]}$ 在 $\alpha$-NPD 和 $\mathrm{Alq}_{3}$ 之间插人一定厚 度的 lithium fluoride $(\mathrm{LiF})$ 插层, 发现 $\mathrm{LiF}$ 插层能明显 增大器件的 MEL 值, 但是其线型并未发生改变(表现 为随磁场的增大先迅速增大, 最后均趋于饱和). 他 们还发现 $\mathrm{LiF}$ 插层的厚度不同, MEL 的最大值也不同, 最大可达到 $8 \%$ (对应插层厚度为 $1.5 \mathrm{~nm}$ ). 在我们以 前的工作中也曾发现类似的现象 ${ }^{[14]}$, 并且我们还进 一步研究了低温下 $(15 \mathrm{~K}) \mathrm{LiF}$ 插层对器件电致发光磁 场效应的影响. 发现插层越厚, 高场下降越小, 当插 层厚度为 $3 \mathrm{~nm}$ 时, MEL 几乎没有出现高场下降. 上 述结果都是基于 $\mathrm{LiF}$ 分子的绝缘性质, 即 $\mathrm{LiF}$ 插层增 大了界面附近电子和空穴的距离, 增强了单重态电 子-空穴对向三重态电子-空穴对的系间苴越(ISC)过 程, 从而增大插层器件的磁效应值. 然而, 上述工作 均未考虑 $\mathrm{LiF}$ 分子在外电场中还具有的极化特性. 文 献[16]报道 $\mathrm{LiF}$ 分子相当于一个电偶极子, 在外电场

引用格式: 白江文, 张巧明, 雷衍连, 等. $\mathrm{LiF}$ 分子的极化对有机发光二极管磁效应的影响. 科学通报, 2013, 58: 3045-3051 Bai J W, Zhang Q M, Lei Y L, et al. The influence of polarization in LiF molecules on the magnetic field effects in OLEDs (in Chinese). Chin Sci Bull (Chin Ver), 2013, 58: 3045-3051, doi: 10.1360/972012-1653 
作用下分子两端将产生极化电荷. 这导致发光器件 中载流子的运输、激子的形成以及激子之间的相互作 用发生改变, 进而为研究有机磁效应的物理机制(特 别是激子与电荷相互作用机制) 提供更为充实的实验 证据.

为了使 $\mathrm{LiF}$ 分子的极化特性更加明显, 并考虑到 $\mathrm{LiF}$ 插层器件的一些缺点 $(\mathrm{LiF}$ 插层的极化特性不显著, 插层对载流子的阻挡作用使得器件注人不平衡等), 我们制备了 $\mathrm{LiF}$ 掺杂器件. 器件结构为: ITO/Copper phthalocyanine $(\mathrm{CuPc})(15 \mathrm{~nm}) / \mathrm{N}, \mathrm{N}^{\prime}$-bis (naphthalen$1-\mathrm{y})-\mathrm{N}, \mathrm{N}^{\prime}$-bis (phenyl) benzidine (NPB) $(60 \mathrm{~nm}) /$ tris(8-hydrox-yquinoline) aluminum (III): lithiumfluoride (LiF) $(60 \mathrm{~nm}, x \%) /$ Bathocuproine (BCP) $(20 \mathrm{~nm}) / \mathrm{LiF}$ $(1 \mathrm{~nm}) / \mathrm{Al}(120 \mathrm{~nm}),(x$ 在 $0 \sim 12 \%$ 之间), 并在不同温 度下研究了不同掺杂浓度器件的 MC 及 MEL. 另外, 本文建立了 $\mathrm{LiF}$ 分子在外电场作用下的极化模型, 并 分析了 $\mathrm{LiF}$ 掺杂影响器件磁效应曲线的原因, 这不仅 能促进对有机发光二极管磁效应物理机制的理解, 同时也提供了一种增大磁效应值的方法, 提高了磁 效应的潜在应用价值.

\section{1 实验}

本文以 $\mathrm{Alq}_{3}$ 为发光层, $\mathrm{CuPc}$ 为空穴注人层, $\mathrm{NPB}$ 为空穴传输层, $\mathrm{BCP}$ 为电子传输层兼作空穴阻挡 层, 以无机离子型晶体 $\mathrm{LiF}$ 为掺杂剂, 制备了 $\mathrm{LiF}$ 掺 杂器件, 其结构如图 1(a)所示. 器件制备过程大致如 下: 首先, 清洗带有 $\mathrm{ITO}$ (作为器件的阳极)的玻璃基 片, 先用 Decon 90 清洗液 (浓度为 4\%)进行水浴超声 清洗(水温加热至 $60^{\circ} \mathrm{C}$ ), 再用无尘纸擦拭 ITO 玻璃, 接着用去离子水、酒精和丙酮分别超声水浴清洗, 清 洗过程中变化超声频率. 其次, 在装样台上将 ITO 基 片从沸腾的丙酮溶液中快速取出, 将其固定在样品 托上, 并快速转移到高真空分子沉积系统真空腔内 (本底真空度 $10^{-7} \mathrm{~Pa}$ ). 然后, 进行有机功能层和电 极的蒸镀. $\mathrm{Alq}_{3}$ 与 $\mathrm{LiF}$ 掺杂层采用共蒸发的方法制得, 掺杂浓度是通过调节 $\mathrm{Alq}_{3}$ 和 $\mathrm{LiF}$ 的热蒸发速率来控 制的. 蒸发速率及薄膜厚度是采用 INFICON 公司的 膜厚检测仪 (XTM/2) 进行原位监测, 速率控制在 $0.05 \sim 0.2 \mathrm{~nm} / \mathrm{s} .120 \mathrm{~nm}$ 厚的 $\mathrm{Al}$ 作为器件的阴极. 有机 层和阴极 $\mathrm{Al}$ 之间的 $\mathrm{LiF}(1 \mathrm{~nm})$ 是为了增强器件的电子 注人效率. ITO、有机层和 $\mathrm{Al}$ 三者之间的交叠部分为 器件的有效发光区域, 面积约为 $1 \mathrm{~mm} \times 2 \mathrm{~mm}$.

测量时, 将样品快速地固定到一套闭循环冷却

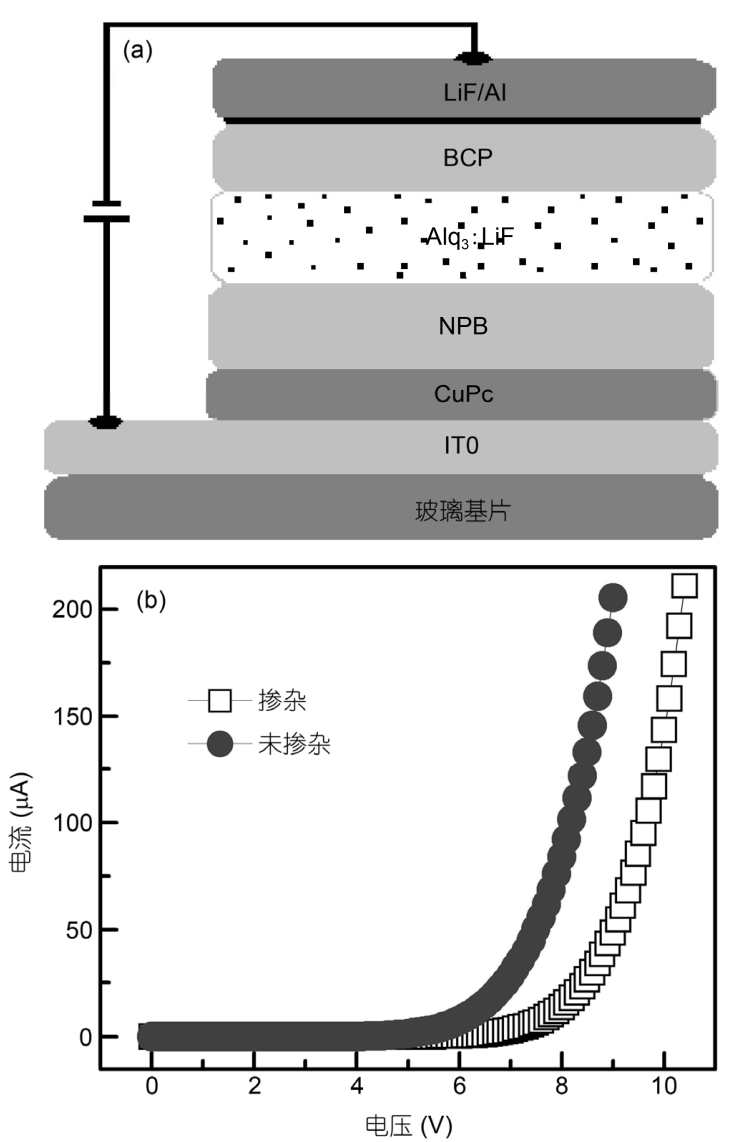

图 1 (a) 器件结构示意图; (b) 室温下 LiF 掺杂器件与未掺 杂器件的电流-电压 $(I-V)$ 特性曲线

系统(Janis: CCS-305S)的冷头上, 其温度在 15 300 K 范围内连续可调. 外部磁场由位于冷却系统两侧的 电磁铁提供, 磁场大小由计算机控制的励磁电源 (Lakeshore EM647)调控, 由 Lakeshore421 高斯计测 量, 最大可达到 $500 \mathrm{mT}$, 磁场方向平行于样品表面 (垂直于电流方向). 器件的驱动电压通过 Keithley 2400 电源提供, 同时还可以测量器件的电流. 器件 的发光强度是由固定在 Janis: CCS-305S 透明外罩上 的光电探测器测得并通过 Keithley 2000 输出. 经过 反复的实验, 我们已经确认, 硅光电探测器的输出本 身不受外磁场的影响, 而且, 外加磁场的方向与流过 器件电流的方向无关, 对实验结果没有任何影响.

\section{2 结果}

\section{1 器件结构及其 $I-V$ 特性曲线}

图 1(a)为器件的结构示意图, 阳极 ITO 和阴极 $\mathrm{Al}$ 之间是各种有机功能层. 图 1(b)为室温下 $\mathrm{LiF}$ 掺杂 
器件与未掺杂的参考器件的电流-电压 $(I-V)$ 特性曲线, 从该图可以看出, 两者都表现出了典型的二极管特 性. 另外, $\mathrm{LiF}$ 掺杂增大了器件的启亮电压, 这与我 们以前关于 $\mathrm{LiF}$ 插层的报道 ${ }^{[14]}$ 相类似. 其他温度下的 电流-电压特性曲线与室温下相似, 只是温度越低, 器件的开启电压越大.

\section{2 室温下 $\mathrm{LiF}$ 掺杂器件注入电流和电致发光的 磁场效应}

图 2(a)和(b)分别给出了室温下 $\mathrm{LiF}$ 掺杂器件与参 考器件的磁电导曲线. 磁电导的定义是: $\mathrm{MC}=[[I(B)-$ $I(0)] / I(0)] \times 100 \%$, 其中 $I(B)$ 和 $I(0)$ 分别为有、无磁场 时流过器件的电流. 从该图可以看出, 与未掺杂器件 的磁电导曲线相比, 掺杂器件的磁电导曲线的线型 发生了较明显的改变. 未掺杂器件的磁电导曲线表 现为随磁场的增加先快速增加(斜率绝对值较大), 然 后增加变缓(斜率绝对值变小)并趋于饱和; 而掺杂器 件的 $\mathrm{MC}$ 在小磁场部分 $(B<40 \mathrm{mT})$ 表现为小幅的快速 增加, 在高场部分 $(B>40 \mathrm{mT})$ 则表现出近乎直线增加, 需要特别指出的是, 掺杂器件的 $\mathrm{MC}$ 在大磁场下没有 表现出饱和迹象. 另外, 掺杂器件 MC 的最大值 (500 mT 处所对应的值)与未掺杂器件相比也明显增
加. 上述现象在以前的文献中未见报道.

此外, 与未掺杂的参考器件相比, 掺杂器件电致 发光的磁效应(MEL)也表现出了线型和值的变化, 如 图 2(c) 和(d)所示. 其中 MEL 的定义与 MC 的定义相 似, 即 $\mathrm{MEL}=[[\mathrm{EL}(B)-\mathrm{EL}(0)] / \mathrm{EL}(0)] \times 100 \%, \mathrm{EL}(B)$ 和 $\operatorname{EL}(0)$ 分别为有、无磁场时器件的电致发光强度. 从 图中可以发现, 掺杂器件的 MEL 曲线的高场也不饱 和. 同时, 其最大值与未掺杂器件的最大值相比也有 较大幅度的增加. 另外, 与参考器件 MEL 的偏压依 赖关系所不同, 掺杂器件的 MEL 曲线在不同偏压下 都重合在一起, 即 MEL 曲线几乎不随器件偏压(电流) 的改变而变化. 总体看来, $\mathrm{LiF}$ 掺杂无论对器件的 $\mathrm{MC}$ 和 MEL 的线型还是值的大小, 都能产生较为明显的 影响.

\section{3 温度对器件磁场效应的影响}

图 3(a) (d) 分别为 $300,200,100,50 \mathrm{~K}$ 下 $\mathrm{LiF}$ 掺杂 器件与参考器件的磁电导曲线. 为了便于比较, 所有 温度下器件的掺杂浓度均为 $8 \%$, 注人电流都在 $100 \mu \mathrm{A}$ 左右. 如图所示, $\mathrm{LiF}$ 掺杂器件的 $\mathrm{MC}$ 最大值随温度 降低而快速减小 $(300 \mathrm{~K}$ 时最大值可达到 $3.6 \%$, 而 $50 \mathrm{~K}$ 时仅仅为 $0.6 \%$ ), 与此不同, 未掺杂器件的 $\mathrm{MC}$

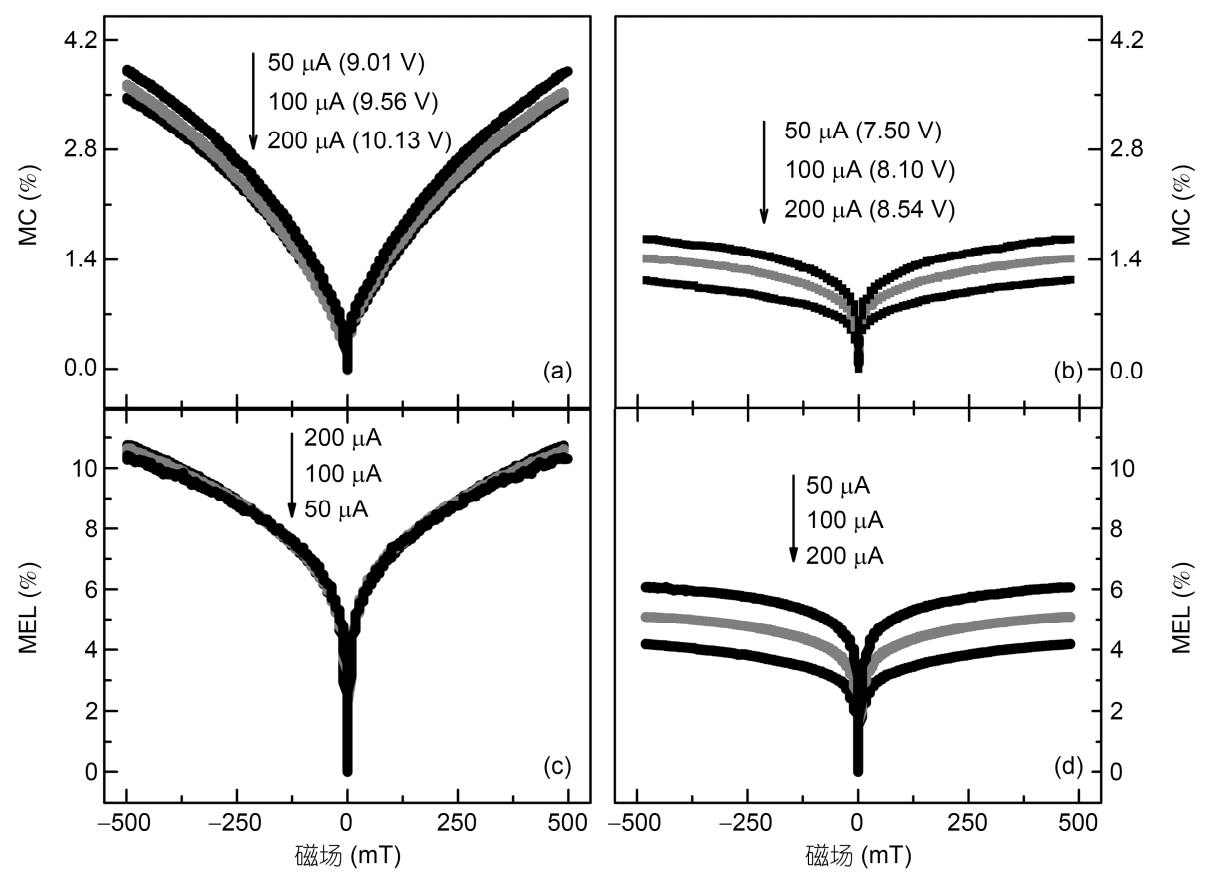

图 2 室温下, 不同偏压(对应不同电流)所对应的器件 MC 和 MEL

(a) 掺杂器件 MC; (b) 未掺杂器件 MC; (c) 掺杂器件 MEL; (d) 未掺杂器件 MEL 
值随温度的变化较小 $(300 \mathrm{~K}$ 时最大值为 $1.4 \%, 50 \mathrm{~K}$ 时为 $0.6 \%$ ). 上述结果表明, $\mathrm{LiF}$ 掺杂不仅能增大器件 的 $\mathrm{MC}$ 值, 而且也使得器件对温度的依赖关系更敏感, 即 $\mathrm{LiF}$ 的引人为提高器件对磁场和温度的灵敏度提 供了可借鉴的方法. 更为重要的是, 在所有温度下 $(50 \sim 300 \mathrm{~K}), \mathrm{LiF}$ 掺杂器件和参考器件的 $\mathrm{MC}$ 的线型都 有较明显的区别, 这一点在图 3(d)中可以看得更清楚. 在图 3(d)中, 两种器件的最大值都约为 $0.6 \%$, 但二者 趋于最大值的方式不同. 当外磁场大于 $40 \mathrm{mT}$ 后, 参 考器件几乎水平趋近于最大值, 而掺杂器件却呈近 似直线增加达到最大值. 从图 3 我们可以更进一步的 理解本文所说的曲线线型不同的含义: 当外磁场大 于 $40 \mathrm{mT}$ 后，两者趋于最大值的途径的不同. 这说明 掺杂改变了器件中影响磁电导的微观机制. 另外, 掺 杂器件的 MC 在所测磁场范围内并未出现饱和的趋 势. 在此情况下, 如果进一步增大磁场, 将有可能获 得更大的 MC 值.

\section{4 掺杂浓度对器件磁效应的影响}

室温下, 掺杂器件 MC 和 MEL 的高场效应(high field effect, HFE)随浓度的变化趋势, 分别如图 4(a), (b)所示. 磁电导的高场效应定义为: HFE=[[I(500 mT)-
$I(40 \mathrm{mT})] / I(0)] \times 100 \%^{[17]}$, 其值的大小表示器件的 MC 值从 $40 \mathrm{mT}$ 到 $500 \mathrm{mT}$ 的变化程度的大小. HFE 值越 大, 变化程度越大, 曲线变化越陡. 从图中可以看到, 掺杂器件 $\mathrm{MC}$ 的高场效应的大小依赖于掺杂浓度, 并 随掺杂浓度的增加表现出先增加后下降的变化规律. 当掺杂浓度为 $6 \%$ 左右时, 磁电导的 HFE 值最大, 掺 杂器件的高场值始终大于未掺杂器件的高场值(浓度 为 0 时的 HFE 的值). 并且, 在不同电流密度的条件 下, 器件的最适掺杂浓度都为 $6 \%$. MEL 的高场效应 随浓度的变化与 MC 相似, 如图 4(b)所示. 此外, 我 们还做了更大掺杂浓度的器件 (由于篇幅限制, 在此 未呈现), 这些器件测量结果显示, 随着浓度的进一 步增加, 磁电导值也进一步降低. 这一现象表明了 $\mathrm{LiF}$ 对器件的磁效应有直接的影响.

\section{3 分析与讨论}

有机发光二极管中，电子和空穴分别从器件的 阴极和阳极注人，经电子传输层和空穴传输层到达 发光层 $\left(\mathrm{Alq}_{3}\right)$, 形成单重态和三重态两种电子-空穴对, 进而形成相应的激子, 数量比为 $1: 3^{[10]}$. 单重态和三 重态电子-空穴对之间可以通过系间鼌越(intersystem crossing, ISC)过程而发生相互转换 ${ }^{[18,19]}$, 从而可以改

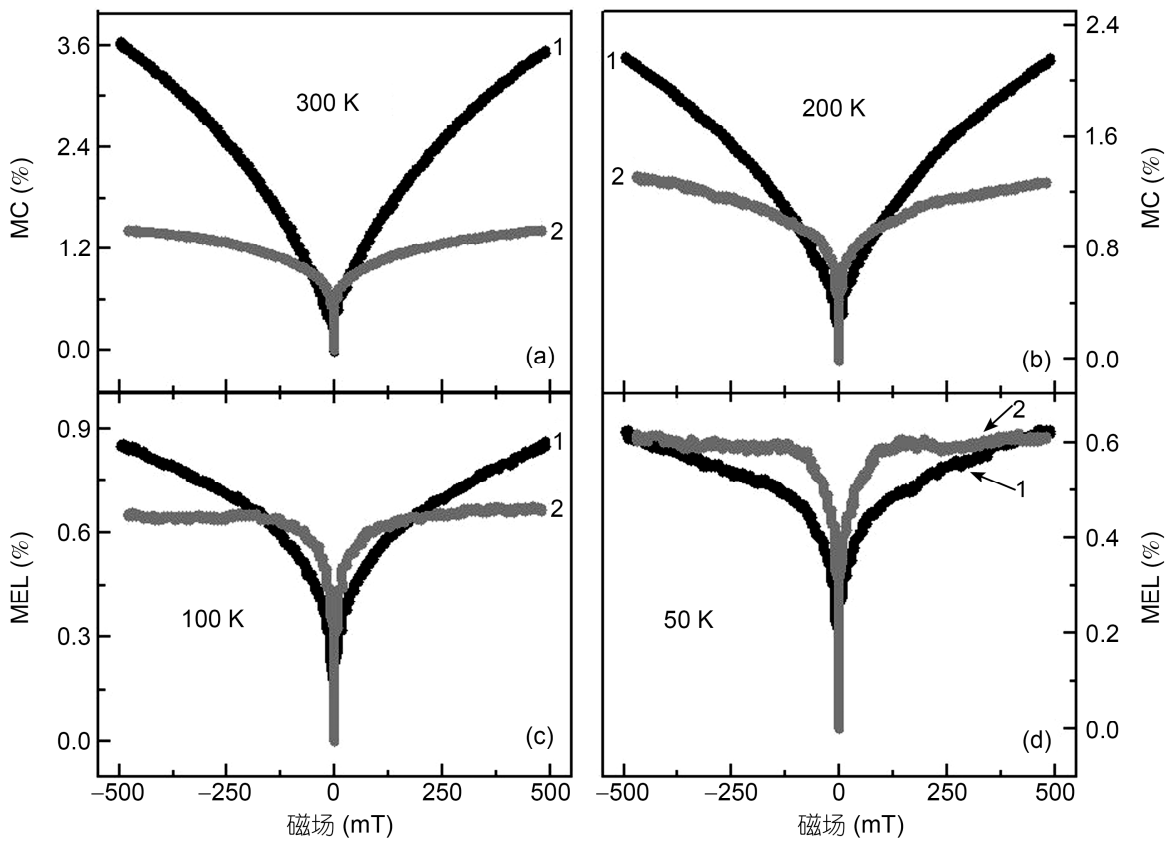

图 3 (a) (d) 分别为 300, 200, 100, 50 K 下掺杂器件与参考器件的磁电导的的变化趋势 电流和掺杂浓度分别为 $100 \mu \mathrm{A}$ 和 $8 \%$. 曲线 1 代表掺杂器件, 曲线 2 代表参考器件 


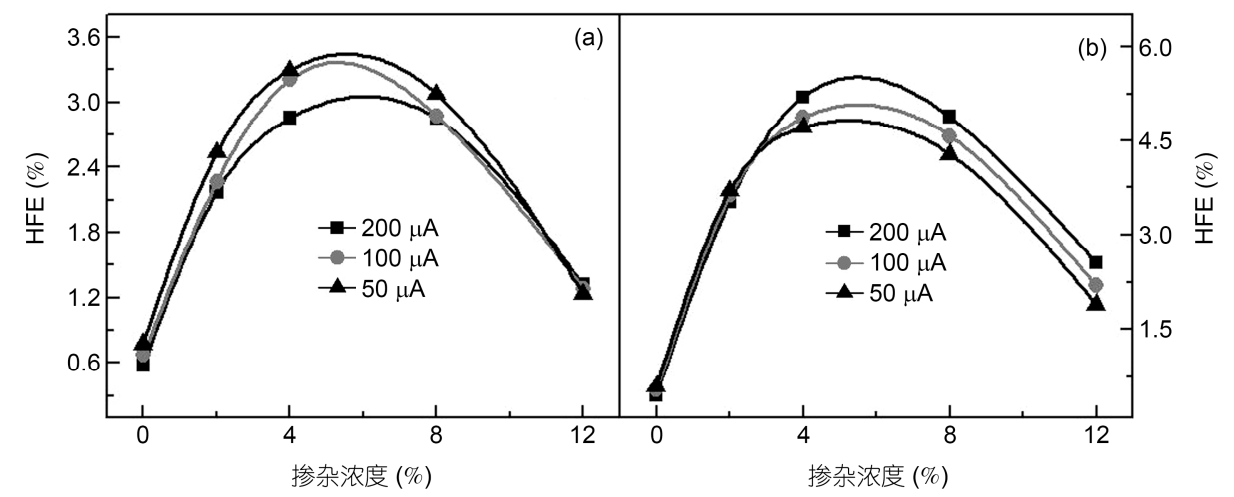

图 4 (a), (b) 分别为室温下掺杂器件的 MC 和 MEL 高场效应随掺杂浓度的变化

变单、三重态电子-空穴对间的数量比. 单重态激子 可以通过辐射退激发光, 而三重态激子(T)由于自旋 禁阻而不能直接发光，只能通过非辐射退激或解离. $\mathrm{T}$ 激子解离成为电子和空穴, 从而增大通过器件的电 流. 此外, 三重态激子还可以和器件中的自由载流子 反应 ${ }^{[6,10,20]}$ (即 TQA), 通过该反应过程, $\mathrm{T}$ 激子被淬灭 到基态 (三重态激子丢失), 通过解离途径对器件电流 产生贡献的 $\mathrm{T}$ 激子的数量减少, 从而影响器件的电 流. 一般认为, ISC 和 TQA 过程都受磁场的抑制 ${ }^{[17,18]}$, 从而使得有机发光器件的电流和发光对外磁场有响 应, 产生磁场效应. 需要指出的是, 三重态激子的寿 命与温度密切相关, 温度越低, 三重态激子的寿命越

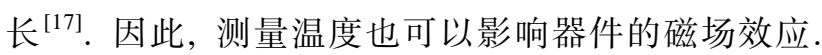
对本文的参考器件而言, 由于注人相对平衡, 器件中 几乎没有剩余电荷的累积, 不易观察到 TQA 过程对 器件磁效应的影响, 其 MC 和 MEL 都表现为随磁场 的增大先快速增大, 而后缓慢增大并趋于饱和, 如图 2(b), (d)所示. 此现象可以用磁场抑制超精细相互作 用进而调节 ISC 过程的速率进行解释 ${ }^{[1,19,21]}$, 并且也 得到了研究人员的广泛认同.

为了进一步认识有机器件内部激发态和电荷之 间相互作用的微观机制，我们在器件的发光层掺人 一定浓度的 $\mathrm{LiF}$, 用以调节器件内部的电荷浓度. 文 献[16]报道, $\mathrm{LiF}$ 为离子型晶体, 其分子相当于一个电 偶极子, 在外电场作用下, 将产生极化电荷. 由此产 生的极化电荷将使得周围的 $\mathrm{Alq}_{3}$ 分子中产生相应的 感应电荷. 由于这些感应电荷不是由注人而来, 因此, 在器件中将造成剩余电荷的产生, 这些剩余电荷将 对器件的 MC 和 MEL 造成较大的影响, 如图 2 所示. 为了便于描述, 我们将磁效应曲线分为低场 $(B<40$ $\mathrm{mT})$ 和高场 $(B>40 \mathrm{mT})$ 两部分, 而且将主要讨论 $\mathrm{LiF}$
对 $\mathrm{MC}$ 的影响. 对磁效应的低场而言, 由 $\mathrm{LiF}$ 引人的 感应电荷主要是降低了其低场值, 而对其线型无影 响. 这是因为, 掺杂的 $\mathrm{LiF}$ 仅仅是淬灭了少量单/三重 态激子, 使得激子解离成的电子和空穴的数量减少, 并不影响单重态和三重态电子-空穴对之间的 ISC 过 程. 对高磁场而言, $\mathrm{LiF}$ 的引人不仅改变了高场的线 型而且也增大了磁效应值. 无磁场时, 由注人形成的 三重态激子与 $\mathrm{LiF}$ 表面的极化电荷以其周围 $\mathrm{Alq}_{3}$ 分 子中的感应电荷发生反应(TQA), 使得三重态激子发 生淬灭 (三重态激子丢失). 当外磁场逐渐增大时, 将 抑制三重态激子与电荷的反应，其结果是导致三重 态激子数量相对增加, 这些三重态激子将通过解离 途径而增大器件的电流. 总体说来, 高磁场的情况下, 器件中的电流将增大. 磁场越大, 对三重态激子与感 应电荷反应的抑制作用越强, 因此, 在 $\mathrm{LiF}$ 掺杂器件 中 MC 不会出现饱和的现象. 掺杂器件的 MEL 较未 掺杂器件也表现出较明显的变化(图 2(c)), 这是由 $\mathrm{MC}$ 的变化所引起的. 此外, 由 MC 引起的 MEL 则几 乎不随外加电压变化, 可能是由于 $\mathrm{LiF}$ 在本文所采用 的偏压范围内可接近完全极化, 所以, 外加电压在几 伏范围内的变化几乎不影响由 $\mathrm{LiF}$ 掺杂引起的 TQA 过程的强度, 而不同偏压下 $\mathrm{MC}$ 的微小差别是由于偏 压增加时导致的电流 $I$ 的增大引起的, 如图 2(a)所 示. 因为偏压增加(电流增加)会导致迁移率的增大, 从而导致磁场引起的电流的改变 $\Delta I$ 也会增加, 但是 当偏压增加(电流增加)时通过器件的电流 $I$ 也会增大. 而且, 电流 $I$ 增加的速度比 $\Delta I$ 增加的速度快, 所以由 $\mathrm{MC}=\Delta I / I$ 可知, 当偏压增加(电流增加)时, $\mathrm{MC}$ 总体表 现出下降趋势.

为了证实上述说法的可能性, 我们还进一步比 较了 $\mathrm{LiF}$ 掺杂器件和参考器件在相同电流、不同温度 
情况下的磁电导效应. 如图 3 所示, 温度越低, 掺杂 器件 $\mathrm{MC}$ 的最大值越小. 这是因为温度降低将会降低 载流子的迁移率，从而产生两方面的影响：一方面， 载流子迁移率的降低会直接导致磁电导的值降低; 另一方面, 载流子迁移率的降低会使得注人的电荷 在发光层积累, 导致空间电荷密度增大. 从而 $\mathrm{LiF}$ 掺 杂引起的那部分感应电荷与三重态激子的反应(TQA) 相对减弱，即注人电荷积累在一定程度上“屏蔽”了 由 $\mathrm{LiF}$ 掺杂引起的 $\mathrm{TQA}$ 作用, 导致 $\mathrm{LiF}$ 掺杂引起的 TQA 相对减弱, 其结果表现为器件 MC 的高场上升 的幅度减小.

由此看来, 对于 $\mathrm{LiF}$ 的掺杂主要影响器件的高场 效应, 为了加深对高场效应的认识以及机理的判断, 我们还测量了不同掺杂浓度器件的高场效应的变化 规律, 如图 4 所示. 当掺杂浓度较低时(浓度小于 $4 \%$ ), $\mathrm{LiF}$ 分子在有机层里的分布密度较小, 在外电场作用 下产生的感应电荷的数量也相对较少, 从而三重态 激子与感生电荷发生作用的几率也较小, TQA 作用 强度较弱. 因此, $\mathrm{MC}$ 的高场效应较小. 随着 $\mathrm{LiF}$ 掺杂 浓度的增大, 感应电荷的密度也增大, 所以发生 TQA 作用的几率也随之增大, 将导致 MC 的高场效 应增大. 因此, 在 $\mathrm{LiF}$ 掺杂中, $\mathrm{MC}$ 的高场上升值将比 未掺杂器件上升值大, 并且在较小掺杂浓度范围内 随着浓度的增加而增大. 随着 $\mathrm{LiF}$ 掺杂浓度的进一步 增大(浓度大于 $6 \%$ ), 相邻 $\mathrm{LiF}$ 分子之间将会发生团聚
作用 ${ }^{[22]}$. 整个 $\mathrm{LiF}$ “分子团”对外显示的电量相对于 未发生团聚作用时会减小, 使得 TQA 作用减弱, 从 而 $\mathrm{MC}$ 的高场上升幅度减小. 此外, 掺杂浓度的增大 将会阻碍器件内载流子的迁移, 从而降低其迁移率. 这样将导致注人电荷在发光层内的积累, 进而屏蔽 $\mathrm{LiF}$ 产生的感应电荷的作用, 最终导致 $\mathrm{MC}$ 高场上升 的降低. 综上所述, $\mathrm{LiF}$ 掺杂器件 $\mathrm{MC}$ 的高场上升值 随着掺杂浓度的增加呈现先增加后减小的变化趋势, 如图 4(a)显示. 当掺杂浓度为 $6 \%$ 左右时, MC 的值达 到最大, 证明此时浓度最适宜, 团聚作用最小. 此外, MEL 与 MC 是成正相关的, 随着掺杂浓度的增加, MEL 也表现出了与 MC 相同的变化趋势, 如图 4(b) 所示. 此结果也佐证了 TQA 反应在该掺杂器件中占 主导地位的可能性.

\section{4 结论}

实验结果显示, 在有机发光器件中掺人 $\mathrm{LiF}$ 不仅 能改变器件在室温下 $\mathrm{MC}$ 和 MEL 的线型, 还使得器 件的磁电导对温度变得较敏感. 并且, 随着掺杂浓度 的增大, MC 和 MEL 的值均呈现先增加后减小的变化 趋势. 因为 $\mathrm{LiF}$ 分子在外电场中会发生极化, 产生大 量感生电荷, 引起了激子-电荷反应, 增大了器件在 室温下的磁效应. $\mathrm{LiF}$ 掺杂对有机发光器件磁效应产 生的影响, 为探究有机半导体材料内部激发态的相 互作用机理提供了一条重要的途径.

\section{参考文献}

1 Kalinowski J, Cocchi M, Virgili D P, et al. Magnetic field effects on emission and current in Alq 3 -based lectroluminescent diodes. Chem Phys Lett, 2003, 380: 710-715

2 Xiong Z H, Wu D, Vardney Z V, et al. Giant magnetoresistance in organic spin-valves. Nature, 2004, 427: 821-824

3 Pang Z Y, Chen Y X, Liu T T, et al. Giant magnetoreisitance in $\mathrm{La}_{0.67} \mathrm{Ca}_{0.33} \mathrm{MnO}_{3} / \mathrm{Alq}_{3} / \mathrm{Co}$ sandwiched- structure organic spin valves. Chin Phys Lett, 2006, 23: 1566-1569

4 Dediu V, Hueso L E, Bergenti I, et al. Room-temperature spintronic effects in Alq 3 -based hybrid devices. Phys Rev B, 2008, 78: 115203

5 Nguyen T D, Sheng Y, Wohlgenannt M, et al. Magnetic field-effects in bipolar, almost hole-only and almost electron-only tris(8-hydroxyquinoline) aluminum devices. Phys Rev B, 2008, 77: 235209

$6 \mathrm{Hu} \mathrm{B}, \mathrm{Wu}$ Y. Tuning magnetoresistance between positive and negative values in organic semiconductors. Nat Mater, 2007, 6: 985-990

7 Bergeson J D, Prigodin V N, Lincoln D M, et al. Inversion of magnetoresistance in organic semiconductor. Phys Rev Lett, 2008, 100: 067201

8 Li F, Xin L Y, Liu S Y, et al. Direct measurement of the magnetic field effects on carrier mobilities and recombination in tri-8-hydroxyquinoline-aluminum based light-emitting diodes. Appl Phys Lett, 2010, 97: 073301

9 Bobbert P A, Nguyen T D, Wohlgenannt M, et al. Bipolaron mechanism for organic magnetoresis-tance. Phys Rev Lett, 2007, 99: 216801

10 Desai P, Shakya P, Kreouzis T, et al. Magnetoresistance and efficiency measurement of Alq -based OLEDs. Phys Rev B, 2007, 75: 094423 
11 Gärsitz C, Mücki A G, Cölle M. Influence of an external magnetic field on the singlet and triplet emissions of tris-(8-hydroxyquinoline) aluminum (III) ( $\left.\mathrm{Alq}_{3}\right)$. J Appl Phys, 2005, 98: 104507

12 Chen P, Lei Y L, Song Q L, et al. magnetoelectroluminescence in tris-(8-hydroxyquinoline) aluminum-based organic light-emitting diode doped with fluorescent dyes. Appl Phys Lett, 2009, 98: 213304

13 Odaka H, Okamoto H, Kawasaki M, et al. Control of magnetic-field effect on electroluminescence in Alq ${ }_{3}$-based organic light emitting diodes. Appl Phys Lett, 2006, 88: 123501

14 刘荣, 张勇, 雷衍连, 等. LiF 插层对有机发光二极管磁场效应的调控. 物理学报, 2009, 59: 4283-4288

15 朱文清, 蒋雪茵, 张志林, 等. 有机电致发光器件中阴极界面修饰的材料、方法及分类. 功能材料, 2004, 35: 272-275

16 侯晓远，李述汤. 金属-有机物界面的偶极层. 物理, 1999, 28: 152-157

17 Zhang Q M, Lei Y L, Xiong Z H, et al. Positive and negative components of magnetoconductance in hole transport limited organic light-emitting diodes. Appl Phys Lett, 2011, 98: 243303

18 Lei Y L, Zhang Y, Liu R, et al. Driving current and temperature dependent magnetic-field modulated electroluminescence in Alq ${ }_{3}$-based organic light emitting diode. Org Electron, 2009, 10: 889-894

19 Davis A H, Bussmann K. Large magnetic field effects in organic light emitting diodes based on tris (8-hydroxyquinoline aluminum) $\left(\mathrm{Alq}_{3}\right) / \mathrm{N}, \mathrm{N}$ '-Di(naphthalene-1-yl)-N, N'diphenyl-benzidine (NPB) bilayers. J Vac Sci Technol A, 2004, 22: 1885-1891

20 张巧明, 陈平, 雷衍连, 等. 空穴阻挡层 BCP 对掺杂型有机发光二极管中磁电导效应的影响. 中国科学: 物理学 力学 天文学, 2010 , 40: $1507-1513$

21 Zhang Y, Liu R, Lei Y L, et al. Low temperature magnetic field effects in $\mathrm{Alq}_{3}$-based organic light emitting diodes. Appl Phys Lett, 2009, 94: 083307-083309

22 Choudhury K R, Yoon J H, So F. LiF as an n-dopant in Tris(8-hydroxyquinoline)Aluminum thin films. Adv Mater, 2008, 20: 1456-1461

\title{
The influence of polarization in LiF molecules on the magnetic field effects in OLEDs
}

\author{
BAI JiangWen ${ }^{1}$, ZHANG QiaoMing ${ }^{1}$, LEI YanLian ${ }^{1}$, ZHANG Yong $^{1} \&$ XIONG ZuHong ${ }^{1,2}$ \\ ${ }^{1}$ School of Physical Science and Technology, MOE Key Laboratory on Luminescence and Real-Time Analysis, Southwest University, Chongqing 400715, \\ China; \\ ${ }^{2}$ Surface Physics Laboratory (National Key Laboratory), Fudan University, Shanghai 200433, China
}

Doping lithium fluoride $(\mathrm{LiF})$ in the fluorescent layer of conventional organic light-emitting diode is able to effectively tune the magnetic field effects (MFEs) on the current and electroluminescence. As compared with the undoped device, the line-shape and amplitude of MFEs in LiF-doped device showed apparent variation. At room temperature, when the applied external magnetic field reached several hundreds mili-Tesla, the MFEs in conventional device slowly increased and tended to be saturated. However, the MFEs in doped device almost linearly increased with the increasing field, displaying the abnormal and unsaturated high-field effects. Additionally, the amplitudes of high-field effects in current and fluorescence all went up firstly and down later when increasing the doping concentration of LiF. The differences between the MFEs in doped and undoped devices can be attributed to the dielectric polarization of $\mathrm{LiF}$ particles in the device's internal electric field. The reaction between the excitons in fluorescent layer and polarization charges on the surface of LiF particles resulted in the special MFEs in doped device.

magnetoconductance, polarization, triplet excition, exciton-charge annihilation

doi: 10.1360/972012-1653 